\title{
Study of the Response Prediction of a Parametric Vibration Based on Modulation Feedback
}

\author{
Dishan Huang \\ Department of Mechanical Engineering and Automation, Shanghai University, 149 Yanchang Road, Shanghai 200072, China \\ Correspondence should be addressed to Dishan Huang, hdishan@shu.edu.cn
}

Received 29 September 2010; Accepted 31 December 2010

Academic Editor: Jorge Arenas

Copyright () 2010 Dishan Huang. This is an open access article distributed under the Creative Commons Attribution License, which permits unrestricted use, distribution, and reproduction in any medium, provided the original work is properly cited.

The problem of response prediction is investigated for parametric vibration in terms of a new concept. The response solution is presented in the special form of Fourier series for signal degree freedom of parametric vibration based on modulation feedback. By applying harmonic balance and limitation operation, all coefficients of a harmonic component are fully determined with a set of series. Meanwhile, some important dynamic behaviors are exposed through mathematical deduction, and an instability phenomenon can be discussed through given frequency factors. The investigation result shows that the new approach has an advantage in the complete response expression, and it is very significant for the theoretical research and engineering application concerning parametric vibration.

\section{Introduction}

The problem of parametric vibration arises in many branches of physics and engineering, and investigation of stability and response prediction are the two most significant dynamic problems in the parametric vibration system. In the past, several methods have been used to study the stability of systems with periodic coefficients. These include Hill's Method [1], the perturbation method [2], the averaging approach, Floquet theory with numerical integration [3], and Sinha's numerical scheme with the shifted Chebyshev polynomial $[4,5]$.

Hill's infinite determinant was used to compute the transition curve or the instability boundary. However, it does not yield the response vector at an arbitrary point in the parametric space. The perturbation method supplies a method for obtaining the solution vector and instability boundary. The solution vector is expanded as a power series in terms of the small parametric and substituted into the original equation. The method is applicable only if the parametric multiplying the period term is small [6]. Authors [7-9] have tried to use the approximate approach to determine the stability and response from an approximate system of equations, which is usually obtained by replacing the elements of the periodic coefficients matrix with piecewise constants or linear functions. Sinha and $\mathrm{Wu}$ presented a numerical scheme for computing the Floquet transition matrix associated with a class of periodic systems. In the authors' approach, the solution vector is expanded in terms of the shifted Chebyshev polynomials. This technique reduces the original differential system to a system of linear algebraic equation from which the solutions in the interval of one period can be easily obtained.

In science and engineering practice, it will be very useful to express the response solution in the form of a Fourier series, which is convenient in an online vibration monitoring system. Especially in a mechanical fault diagnosis, such expression is important and not replaceable. One typical example is to diagnose the mechanical fault in a rotational rotor with a cross crack by analyzing a characteristic of the parametric system [10]. However, so far, no related response solution has directly been considered in all of the above approaches.

In this paper, the concept of modulation feedback is introduced to set a response form that is similar to a Fourier series. Using harmonic balance in the equation and limitation operation, a full response expression is 


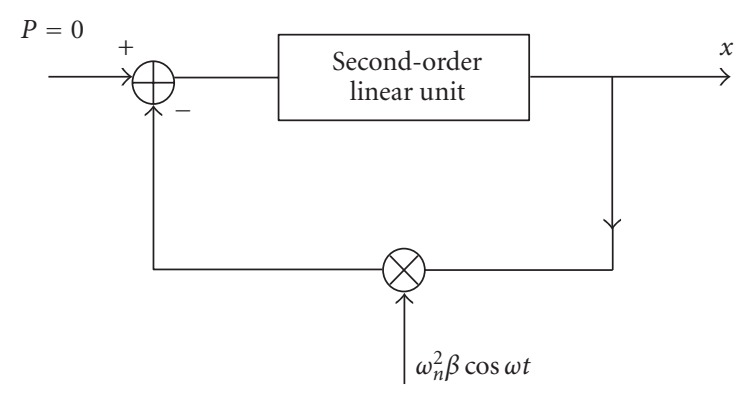

FIgURE 1: Modulation feedback system.

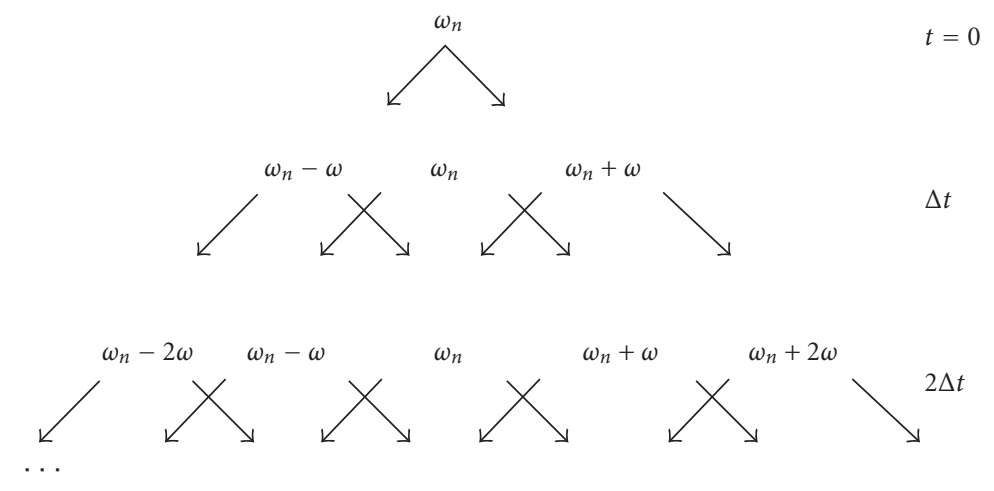

FIGURE 2: Frequency fission procedure $(t \geq 0, \Delta t \rightarrow 0)$.

conducted. Though this concept comes from the content of a modulation system, it reflects the intrinsic physical character of a parametric system. Moreover, the presented solution conducts the response prediction that could be seen as an expansion of the Perturbation solution.

\section{Modulation Feedback Conception}

Consider a classical parametric vibration equation:

$$
\frac{d^{2} x}{d t^{2}}+\omega_{n}^{2}(1+\beta \cos \omega t) x=0,
$$

and rewrite it as

$$
\frac{d^{2} x}{d t^{2}}+\omega_{n}^{2} x=-x \omega_{n}^{2} \beta \cos \omega t
$$

Based on (2), the problem of parametric vibration can be schematically described as a system drawn in Figure 1, and it is a special feedback system that contains a second-order linear unit and a frequency modulation. The output of the system is the general solution of parametric vibration.

In this system, in addition to enhancing the input and feedback signal, the second-order linear unit will inherently generate the harmonic component $C_{01} \cos \omega_{n} t$ after the moment $t=0$. However, the generated component $C_{01} \cos \omega_{n} t$ will be multiplied by $\omega_{n}^{2} \beta \cos \omega t$, and this operation is called the frequency modulation, which conducts frequency fission in the system.
The phenomenon of frequency fission can be stated as the following: the component $\omega_{n}$ is fissile into the combination of harmonic components $\omega_{n}-\omega$ and $\omega_{n}+\omega$ by a modulation operation within the first $\Delta t$. Then, the modulation result directly feeds back to the second-order linear unit as an input of the new harmonic components. Thus, within the second $\Delta t$, three components $\omega_{n}, \omega_{n}-\omega$, and $\omega_{n}+\omega$ will be the output of the system and be involved in another fission operation, a sequential combination of five harmonic components $\omega_{n}, \omega_{n}-\omega, \omega_{n}-2 \omega, \omega_{n}+\omega$, and $\omega_{n}+2 \omega$ will become the modulation results and will feed back to the second-order linear unit, and the iterate fission operation continues. The whole physical procedure of the frequency fission is schematically described in Figure 2.

Assuming the frequency fission procedure continues, except for the case of the instable state, the frequency fission will attain a state of balance. Therefore, there are many linear combinations of harmonic components related to $\omega_{n}$ and $\omega$ in the output of the system as the frequency fission balance, and they can be mathematically expressed as follows:

$$
x=\sum_{k=-\infty}^{\infty} C_{k} \cos \left(\omega_{n}+k \omega\right) t
$$

Because the energy of the harmonic components is concentrated on the narrow band range in the frequency domain, the coefficient $C_{k} \rightarrow 0$ while $k \rightarrow \infty$. 
Therefore, the response solution problem of the parametric vibration equation is simplified to the determination of coefficient values of $C_{k}$ in (3).

\section{Fission Coordinate Equation}

After substituting the solution (3) into (1), we obtain the following infinite set of linear, algebraic, homogeneous equations for the $C_{k}$ by equating the coefficients of each harmonic on both sides of (1):

$$
\begin{gathered}
\frac{\omega_{n}^{2} \beta}{2} C_{k-1}+\left[\omega_{n}^{2}-\left(\omega_{n}+k \omega\right)^{2}\right] C_{k}+\frac{\omega_{n}^{2} \beta}{2} C_{k+1}=0 \\
(k=\cdots-m,-(m-1), \ldots,-3,-2 \\
-1,0,1,2,3 \cdots m-1, m \cdots) .
\end{gathered}
$$

Here, we introduce notations:

$$
\begin{aligned}
\omega_{k} & =\omega_{n}^{2}-\left(\omega_{n}+k \omega\right)^{2} \\
& (k=\cdots-m,-(m-1), \ldots,-3,-2,-1, \\
& 0,1,2,3 \cdots m-1, m \cdots), \\
\gamma & =\frac{\omega_{n}^{2} \beta}{2} .
\end{aligned}
$$

First, we consider $2 m+1$ finite equations, and put all coefficient relations together and form a linear algebra equation called a fission coordinate equation (which is similar to Hill's form).

$$
\left[\begin{array}{cccccc}
\omega_{-m} & \gamma & & & \\
\gamma & \omega_{-m+1} & \gamma & & \\
& \cdot & \cdot & \cdot & \\
& & & \gamma & \omega_{-3} & \gamma \\
& & & \gamma & \omega_{-2} \\
& & & & & \\
& & & & & \\
& & & & \\
& & & & \\
& & & & \\
& & & & &
\end{array}\right.
$$$$
\begin{array}{lll}
\gamma & \omega_{-2} & \gamma
\end{array}
$$$$
\begin{array}{lll}
\gamma & \omega_{-1} & \gamma
\end{array}
$$$$
\begin{array}{lll}
\gamma & 0 & \gamma
\end{array}
$$$$
\gamma \omega_{1} \gamma
$$$$
\begin{array}{lll}
\gamma & \omega_{2} & \gamma
\end{array}
$$$$
\begin{array}{lll}
\gamma & \omega_{3} & \gamma
\end{array}
$$$$
\left[\begin{array}{c}
C_{-m} \\
C_{-m+1} \\
\cdot \\
C_{-3} \\
C_{-2} \\
C_{-1} \\
C_{0} \\
C_{1} \\
C_{2} \\
C_{3} \\
\cdot \\
C_{m-1} \\
C_{m}
\end{array}\right]=\left[\begin{array}{c}
-\gamma C_{-(m+1)} \\
0 \\
\cdot \\
0 \\
0 \\
0 \\
0 \\
0 \\
0 \\
0 \\
\cdot \\
0 \\
-\gamma C_{m+1}
\end{array}\right] .
$$

\section{Determination of Coefficient $C_{k}$}

Let frequency factors be

To get a full response expression, the determination of coefficient $C_{k}$ is carried out by the following operation.

(a) Consider the lower half of (6):

$$
\left[\begin{array}{cccccc}
\omega_{1}-\omega_{-1} & \gamma & & & \\
\gamma & \omega_{2} & \gamma & & & \\
& \gamma & \omega_{3} & \gamma & & \\
& & \cdot & \cdot & \cdot & \\
& & & & \gamma & \omega_{m}
\end{array}\right]\left[\begin{array}{c}
C_{1} \\
C_{2} \\
C_{3} \\
\cdot \\
C_{m}
\end{array}\right]=\left[\begin{array}{c}
-\gamma\left(2 C_{0}+C_{-2}\right) \\
0 \\
0 \\
\cdot \\
-\gamma C_{m+1}
\end{array}\right] .
$$

$$
\begin{aligned}
\alpha_{1} & =\omega_{1}-\omega_{-1}, \\
\alpha_{2} & =\omega_{2} \frac{-\gamma^{2}}{\alpha_{1}}, \\
\vdots & \\
\alpha_{m} & =\omega_{m} \frac{-\gamma^{2}}{\alpha_{(m-1)}} .
\end{aligned}
$$


Then

$$
\begin{aligned}
& {\left[\begin{array}{cccccc}
\alpha_{1} & \gamma & & & & \\
& \alpha_{2} & \gamma & & & \\
& & \alpha_{3} & \gamma & & \\
& & \cdot & \cdot & \cdot & \\
& & & & & \alpha_{m}
\end{array}\right]\left[\begin{array}{c}
C_{1} \\
C_{2} \\
C_{3} \\
\cdot \\
C_{m}
\end{array}\right]} \\
& {\left[\begin{array}{c}
-\gamma\left(2 C_{0}+C_{-2}\right) \\
\frac{\gamma^{2}\left(2 C_{0}+C_{-2}\right)}{\alpha_{1}} \\
\frac{-\gamma^{3}\left(2 C_{0}+C_{-2}\right)}{\alpha_{1} \alpha_{2}} \\
\cdot(-1)^{m} \frac{\gamma^{m}\left(2 C_{0}+C_{-2}\right)}{\prod_{i=1}^{m-1} \alpha_{i}}-\gamma C_{m+1}
\end{array}\right]}
\end{aligned}
$$

One of the solutions to (9):

$$
\begin{aligned}
C_{1}=\left(2 C_{0}+C_{-2}\right) \\
\quad \times\left(-\frac{\gamma}{\alpha_{1}}-\frac{\gamma^{3}}{\alpha_{1}^{2} \alpha_{2}}-\frac{\gamma^{5}}{\alpha_{1}^{2} \alpha_{2}^{2} \alpha_{3}}-\cdots\right. \\
\left.\quad-\frac{\gamma^{2 k-1}}{\left(\prod_{i=1}^{k-1} \alpha_{i}^{2}\right) \alpha_{k}}-\cdots+(-1)^{m+2} \frac{\gamma^{m}}{\prod_{i}^{m-1} \alpha_{i}} C_{m+1}\right)
\end{aligned}
$$

As mentioned in the above section, $C_{m+1} \rightarrow 0$ while $m \rightarrow \infty$. Let $k=n$; thus the coefficient $C_{1}$ can be expanded as

$$
C_{1}=\left(2 C_{0}+C_{-2}\right) S_{1},
$$

where

$$
S_{1}=-\frac{\gamma}{\alpha_{1}}-\frac{\gamma^{3}}{\alpha_{1}^{2} \alpha_{2}}-\frac{\gamma^{5}}{\alpha_{1}^{2} \alpha_{2}^{2} \alpha_{3}}-\cdots-\frac{\gamma^{2 n-1}}{\left(\prod_{i=1}^{n-1} \alpha_{i}^{2}\right) \alpha_{n}}-\cdots
$$

For the same reason, $C_{2}, C_{3}, \ldots C_{k}$ can be determined. They are

$$
\begin{aligned}
C_{2} & =\left(2 C_{0}+C_{-2}\right) S_{2}, \\
C_{3} & =\left(2 C_{0}+C_{-2}\right) S_{3}, \\
& \vdots \\
C_{k} & =\left(2 C_{0}+C_{-2}\right) S_{k},
\end{aligned}
$$

where

$$
\begin{aligned}
S_{2}= & \frac{\gamma^{2}}{\alpha_{1} \alpha_{2}}+\frac{\gamma^{4}}{\alpha_{1} \alpha_{2}^{2} \alpha_{3}}+\frac{\gamma^{6}}{\alpha_{1} \alpha_{2}^{2} \alpha_{3}^{2} \alpha_{4}} \\
& +\cdots+\frac{\gamma^{2 n}}{\left(\prod_{i=2}^{n} \alpha_{i}^{2}\right) \alpha_{1} \alpha_{n+1}}-\cdots, \\
S_{3}= & -\frac{\gamma^{3}}{\alpha_{1} \alpha_{2} \alpha_{3}}-\frac{\gamma^{5}}{\alpha_{1} \alpha_{2} \alpha_{3}^{2} \alpha_{4}}-\frac{\gamma^{7}}{\alpha_{1} \alpha_{2} \alpha_{3}^{2} \alpha_{4}^{2} \alpha_{5}} \\
& -\cdots-\frac{\gamma^{2 n+1}}{\left(\prod_{i=3}^{n+1} \alpha_{i}^{2}\right) \alpha_{1} \alpha_{2} \alpha_{n+2}}-\cdots, \\
\vdots & \quad-1)^{k} \sum_{n=1}^{\infty} \frac{\gamma^{2 n+k-2}}{\left(\prod_{j=1}^{k-1} \alpha_{j}\right)\left(\prod_{i=k}^{n+k-2} \alpha_{i}^{2}\right) \alpha_{n+k-1}} .
\end{aligned}
$$

(b) Consider the upper half of (6):

$$
\left.\begin{array}{c}
{\left[\begin{array}{cccc}
\alpha_{-1} & \gamma & & \\
& \alpha_{-2} & \gamma & \\
& \alpha_{-3} & \gamma & \\
\cdot & \cdot & \cdot \\
& & \alpha_{-m}
\end{array}\right]\left[\begin{array}{c}
C_{-1} \\
C_{-2} \\
C_{-3} \\
\cdot \\
C_{-m}
\end{array}\right]} \\
\frac{-\gamma\left(2 C_{0}+C_{2}\right)}{\alpha^{2}\left(2 C_{0}+C_{2}\right)} \\
\frac{-\gamma^{3}\left(2 C_{0}+C_{2}\right)}{\alpha_{1} \alpha_{2}} \\
(-1)^{m} \frac{\gamma^{m}\left(2 C_{0}+C_{2}\right)}{\prod_{i=1}^{m-1} \alpha_{i}}-\gamma C_{-(m+1)}
\end{array}\right],
$$

where the frequency factors

$$
\alpha_{-1}=\omega_{-1}-\omega_{1},
$$

$$
\alpha_{-2}=\omega_{-2} \frac{-\gamma^{2}}{\alpha_{-1}}
$$

$$
\alpha_{-m}=\omega_{-m} \frac{-\gamma^{2}}{\alpha_{-(m-1)}} .
$$

The solution of (17) is

$$
\begin{aligned}
C_{-1} & =\left(2 C_{0}+C_{2}\right) R_{1}, \\
C_{-2} & =\left(2 C_{0}+C_{2}\right) R_{2}, \\
C_{-3} & =\left(2 C_{0}+C_{2}\right) R_{3}, \\
& \vdots \\
C_{-k} & =\left(2 C_{0}+C_{2}\right) R_{k},
\end{aligned}
$$


where

$$
\begin{aligned}
R_{1}= & -\frac{\gamma}{\alpha_{-1}}-\frac{\gamma^{3}}{\alpha_{-1}^{2} \alpha_{-2}}-\frac{\gamma^{5}}{\alpha_{-1}^{2} \alpha_{-2}^{2} \alpha_{-3}} \\
& -\cdots-\frac{\gamma^{2 n-1}}{\left(\prod_{i=1}^{n-1} \alpha_{-i}^{2}\right) \alpha_{-n}}-\cdots, \\
R_{2}= & \frac{\gamma^{2}}{\alpha_{-1} \alpha_{-2}}+\frac{\gamma^{4}}{\alpha_{-1} \alpha_{-2}^{2} \alpha_{-3}}+\frac{\gamma^{6}}{\alpha_{-1} \alpha_{-2}^{2} \alpha_{-3}^{2} \alpha_{-4}} \\
& +\cdots+\frac{\gamma^{2 n}}{\left(\prod_{i=2}^{n} \alpha_{-i}^{2}\right) \alpha_{-1} \alpha_{-(n+1)}}-\cdots, \\
R_{3}= & -\frac{\gamma^{3}}{\alpha_{-1} \alpha_{-2} \alpha_{-3}}-\frac{\gamma^{5}}{\alpha_{-1} \alpha_{-2} \alpha_{-3}^{2} \alpha_{-4}}-\frac{\gamma^{7}}{\alpha_{-1} \alpha_{-2} \alpha_{-3}^{2} \alpha_{-4}^{2} \alpha_{-5}} \\
& -\cdots-\frac{\gamma^{2 n+1}}{\left(\prod_{i=3}^{n+1} \alpha_{-i}^{2}\right) \alpha_{-1} \alpha_{-2} \alpha_{-(n+2)}}-\cdots, \\
\vdots & \\
R_{k}= & (-1)^{k} \sum_{n=1}^{\infty} \frac{\gamma^{2 n+k-2}}{\left(\prod_{j=1}^{k-1} \alpha_{-j}\right)\left(\prod_{i=k}^{n+k-2} \alpha_{-i}^{2}\right) \alpha_{-(n+k-1)}}
\end{aligned}
$$

From (11), (13), (19), and (20), we obtain

$$
\begin{gathered}
C_{-2}=-2 C_{0}\left(1+\frac{R_{1}}{S_{2} R_{1}+S_{1}}\right), \\
C_{2}=-2 C_{0}\left(1+\frac{S_{1}}{R_{2} S_{1}+R_{1}}\right) .
\end{gathered}
$$

The final solution form of (4) is

$$
\begin{aligned}
C_{-k} & =-2 C_{0} \frac{S_{1}}{R_{2} S_{1}+R_{1}} R_{k}, \quad(k=1,2,3, \ldots), \\
C_{k} & =-2 C_{0} \frac{R_{1}}{S_{2} R_{1}+S_{1}} S_{k}, \quad(k=1,2,3, \ldots) .
\end{aligned}
$$

\section{Special Case}

While $\omega=2 \omega_{n} / q$ ( $q$ is an integral number) and $\beta>0$, the response solution (3) is changed to

$$
x=\sum_{k=-\infty}^{\infty} C_{k} \cos (q+2 k) \frac{\omega_{n}}{q} t
$$

If $q$ is an even number, one gets

$$
\begin{aligned}
x= & \sum_{k=-\infty}^{-(1+q / 2)} C_{k} \cos (q+2 k) \frac{\omega_{n} t}{q}+\sum_{k=-q / 2}^{\infty} C_{k} \cos (q+2 k) \frac{\omega_{n} t}{q} \\
= & C_{-(q / 2+1)} \cos \frac{2 \omega_{n} t}{q}+C_{-(q / 2+2)} \cos \frac{4 \omega_{n} t}{q} \\
& +C_{-(q / 2+3)} \cos \frac{6 \omega_{n} t}{q}+\cdots \\
& +C_{-q / 2}+C_{-q / 2+1} \cos \frac{2 \omega_{n} t}{q}+C_{-q / 2+2} \cos \frac{4 \omega_{n} t}{q} \\
& +C_{-q / 2+3} \cos \frac{6 \omega_{n} t}{q}+\cdots \\
= & C_{-q / 2}+\sum_{m=1}^{\infty}\left(C_{-q / 2-m}+C_{-q / 2+m}\right) \cos \frac{2 m \omega_{n} t}{q} .
\end{aligned}
$$

If $q$ is an odd number, one gets

$$
\begin{aligned}
x= & \sum_{k=-\infty}^{-(q+1) / 2} C_{k} \cos (q+2 k) \frac{\omega_{n} t}{q} \\
& +\sum_{k=-(q-1) / 2}^{\infty} C_{k} \cos (q+2 k) \frac{\omega_{n} t}{q} \\
= & C_{-(q+1) / 2} \cos \frac{\omega_{n} t}{q}+C_{-(q+1) / 2-1} \cos \frac{3 \omega_{n} t}{q} \\
& +C_{-(q+1) / 2-2} \cos \frac{5 \omega_{n} t}{q}+\cdots \\
& +C_{-(q-1) / 2} \cos \frac{\omega_{n} t}{q}+C_{-(q-1) / 2+1} \cos \frac{3 \omega_{n} t}{q} \\
& +C_{-(q-1) / 2+2} \cos \frac{5 \omega_{n} t}{q}+\cdots \\
= & \sum_{m=0}^{\infty}\left(C_{-(q+1) / 2-m}+C_{-(q-1) / 2+m}\right) \cos \frac{(2 m+1) \omega_{n} t}{q} .
\end{aligned}
$$

(a) There is a direct component $C_{-q / 2}$ in the response $x$ while $q$ is an even number in (29). This is a kind of particular characteristic exposed by the given approach in the response study of parametric vibration.

(b) Based on (30) and (31), the theoretical spectrum of the response of the parametric vibration can be easily predicted.

(c) While $m=q / 2$ in (30) or $m=(q-1) / 2$ in (31), if the coefficients

$$
C_{0}+C_{-q} \gg C_{0}
$$

the response will be the case of the instability state. 


\section{Complete Form of the Solution}

6.1. Complete Form of the Solution. According to the theory of second-order differential equation, the strict form of a general solution for the given parametric vibration equation should be

$$
x=\sum_{k=-\infty}^{\infty} C_{k} \cos \left(\omega_{n}+k \omega\right) t+D_{k} \sin \left(\omega_{n}+k \omega\right) t .
$$

Meanwhile, it needs to meet the sufficient and necessary condition that the Wronskian determinant is equal to the constant.

In fact,

$$
\frac{d w(t)}{d t}=\frac{d}{d t}\left|\begin{array}{ll}
u_{1}(t) & u_{2}(t) \\
\dot{u}_{1}(t) & \dot{u}_{2}(t)
\end{array}\right|=0
$$

$w(t)$ has no relation with time $t$, where

$$
\begin{aligned}
& u_{1}(t)=\sum_{k=-\infty}^{\infty} C_{k} \cos \left(\omega_{n}+k \omega\right) t, \\
& u_{2}(t)=\sum_{k=-\infty}^{\infty} D_{k} \sin \left(\omega_{n}+k \omega\right) t .
\end{aligned}
$$

Because $w(t)$ is a constant, (33) is the complete form of the general solution of the given parametric vibration equation, where $C_{0}$ and $D_{0}$ are the arbitrary constants in the solution in (33) and depend on the initial condition.

6.2. Determination of Coefficient $D_{k}$. The coefficient $D_{k}$ is determined by using the harmonic balance as the determination of the coefficient value of $C_{k}$ :

$$
\begin{gathered}
D_{-k}=-2 D_{0} \frac{S_{1}}{R_{2} S_{1}+R_{1}} R_{k}, \\
D_{k}=-2 D_{0} \frac{R_{1}}{S_{2} R_{1}+S_{1}} S_{k} .
\end{gathered}
$$

Thus, in the special case of $\omega=2 \omega_{n} / q$, if $q$ is an even number, the complete form of the solution will be

$$
\begin{aligned}
x= & C_{-q / 2}+\sum_{m=1}^{\infty}\left(C_{-q / 2-m}+C_{-q / 2+m}\right) \cos \frac{2 m \omega_{n} t}{q}+D_{-q / 2} \\
& +\sum_{m=1}^{\infty}\left(-D_{-q / 2-m}+D_{-q / 2+m}\right) \sin \frac{2 m \omega_{n} t}{q}
\end{aligned}
$$

If $q$ is an odd number, the complete form of the solution will be

$$
\begin{aligned}
x= & \sum_{m=0}^{\infty}\left(C_{-(q+1) / 2-m}+C_{-(q-1) / 2+m}\right) \cos \frac{(2 m+1) \omega_{n} t}{q} \\
& +\sum_{m=0}^{\infty}\left(-D_{-(q+1) / 2-m}+D_{-(q-1) / 2+m}\right) \sin \frac{(2 m+1) \omega_{n} t}{q} .
\end{aligned}
$$

6.3. Initial Condition. Equation (33) is the parametric vibration solution after the system attains a state of balance through frequency fission. However, when $t=0$, equation (1) degenerates to a linear equation. Therefore, the coefficients $C_{0}$ and $D_{0}$ in (33) can be determined by

$$
C_{0}=\left.x\right|_{t=0}, \quad D_{0}=\left.\frac{1}{\omega_{n}} \frac{d x}{d t}\right|_{t=0} .
$$

6.4. Comparison with Another Method. If $k=-1,0,1$ in (33), we obtain

$$
x=\sum_{k=-1}^{1} C_{k} \cos \left(\omega_{n}+k \omega\right) t+D_{k} \sin \left(\omega_{n}+k \omega\right) t .
$$

This is a form of the parametric vibration solution conducted by singular Perturbation called the two variables expansion. Thus, the presented method conducts the response solution that is the expansion of the solution obtained by Perturbation.

In fact, equation (6) is similar to Hill's form, which was used in the study of the instability boundary of parametric vibration during that time [11]. However, this paper determines the coefficients of the harmonic series by using a special property of $\bar{\omega}_{0}=0$ in (6).

\section{Instability}

There are infinite terms of frequency factor $\alpha_{k}(k=$ $\ldots,-3,-2,-1,1,2,3, \ldots)$ in the expression of the obtained solution, which causes instability in the system while $\alpha_{k}=0$. The frequency factors $\alpha_{k}$ are related to $\omega$ and $\beta$, and the roots of $\alpha_{k}=0$ distribute around the instability areas, which are summarized as follows.

7.1. Principal Instability Area. While $\omega=2 \omega_{n}$ and $k=-1$ are selected, the frequency factor $\alpha_{-1}=\omega_{n}^{2}-\left.\left(\omega_{n}-\omega\right)^{2}\right|_{\omega=2 \omega_{n}}=0$. As a result, the values of $\gamma / \alpha_{-1}$ and $\gamma^{3} / \alpha_{-1}^{2} \alpha_{-2}$ in (23) will tend to be infinite while $\beta \neq 0$. This means the harmonic response corresponding to $C_{-1}$ tends to be infinite in the principal instability area.

7.2. High-Order Instability Boundary Curve. Based on the roots of $\alpha_{k}=\omega_{n}^{2}-\left(\omega_{n}+k \omega\right)^{2}-\gamma^{2} / \alpha_{k+1}=0(k<-1)$, the group of harmonic resonance (higher-order instability) boundary curves are drawn in the parameter plan of $\omega$ and $\beta$, as shown in Figures 3(a)-3(d) (generated by commercial software Maple 9.5), which have the following features.

Left Bias. Due to the term $\gamma^{2} / \alpha_{k+1}$ having influence on frequency factor $\alpha_{k}$, each group of multiple harmonic resonance curves is under bias toward $2 \omega_{n} / m(m=2,3,4, \ldots$, and $k=-2,-3,-4, \ldots)$. In other words, each harmonic resonance boundary curve is located at the left side of the point $2 \omega_{n} / m$ that is the high-order instability point while $\beta$ is close to 0 .

Instability Region Overlap. There are different order harmonic resonance boundary curves in each instability area 


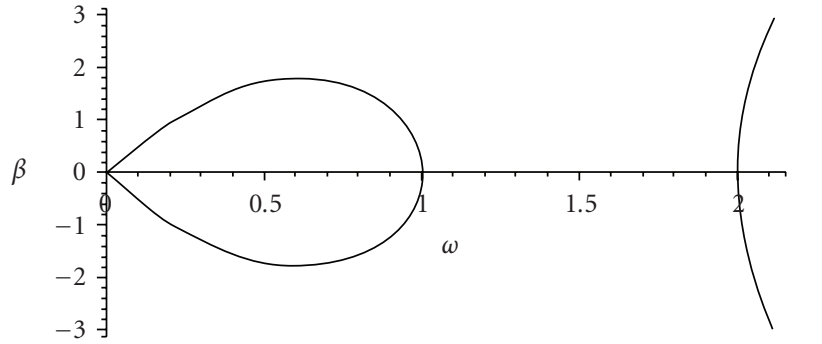

(a) Parameter plan of $\omega$ and $\beta$ while $\alpha_{-2}=0$

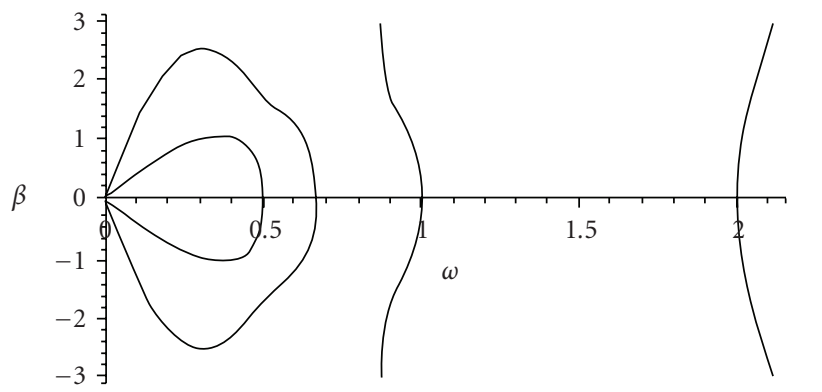

(c) Parameter plan of $\omega$ and $\beta$ while $\alpha_{-4}=0$

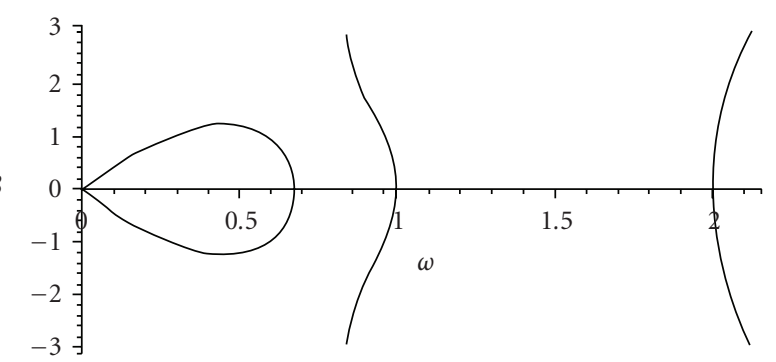

(b) Parameter plan of $\omega$ and $\beta$ while $\alpha_{-3}=0$

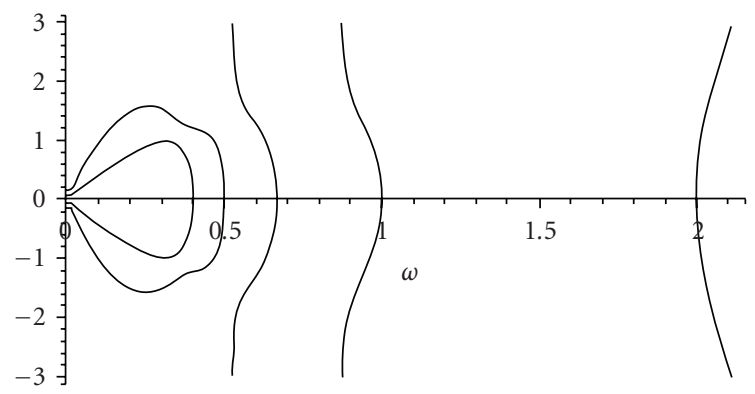

(d) Parameter plan of $\omega$ and $\beta$ while $\alpha_{-5}=0$

FIgURE 3: High-order instability boundary curve.

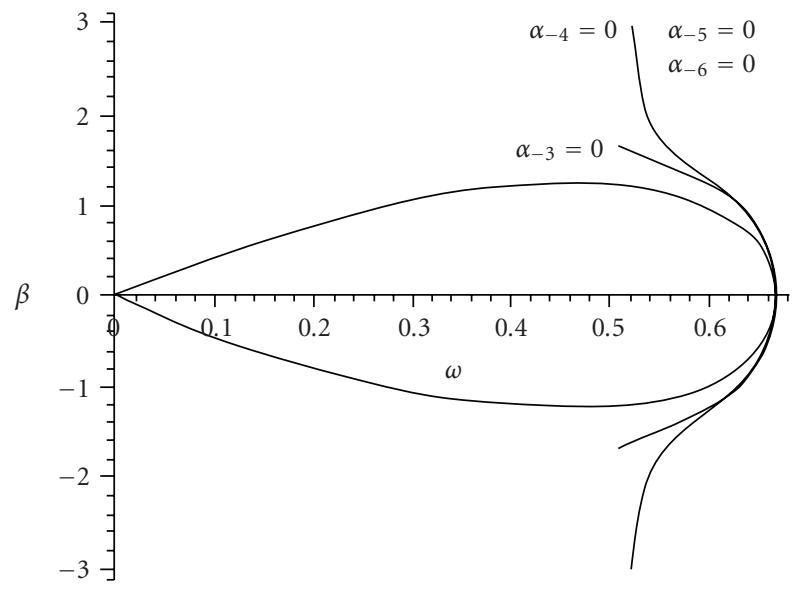

FIgURE 4: Third instability region and boundary curve.

around $2 \omega_{n} / m(m=1,2,3, \ldots)$. For example, as shown in Figure 4, the multiple harmonic resonance regions exit near the third-order instability area, and they are based on $\alpha_{-3}=0, \alpha_{-4}=0 \cdots$; but for $\alpha_{-4}=0, \alpha_{-5}=0 \cdots$ almost overlap in the same region while $0<\beta<1$. Wherein the curve $\alpha_{-3}=0$ is the left side of the instability region, and the curve $\alpha_{-k}=0$ ( $k \rightarrow \infty$, in theory $)$ is the right side of the instability region. Therefore, the harmonic resonance boundary curves $\left(\alpha_{-k}=0\right)$ are composed of the boundaries of the instability region.

Cross Direction. As known from (26), the harmonic resonance strength is different between $\alpha_{k} \rightarrow 0$ and $\alpha_{k+1} \rightarrow 0$. Thus, the harmonic resonance strength is sensitive to the $\omega$ cross direction (from left to right or from right to left) to the high-order instability area.

\section{Example}

Let $\omega_{n}=1, \omega=0.5$, and $\beta=0.3$ in (1), the initial conditions are $\left.x\right|_{t=0}=1$ and $d x /\left.d t\right|_{t=0}=0$.

From (37), the response solution can be expressed as

$$
\begin{aligned}
x(t)= & C_{-2}+\left(C_{-3}+C_{-1}\right) \cos 0.5 t+\left(C_{-4}+C_{0}\right) \cos t \\
& +\left(C_{-5}+C_{1}\right) \cos 1.5 t+\left(C_{-6}+C_{2}\right) \cos 2 t+\cdots
\end{aligned}
$$

On the initial condition, obtain $C_{0}=1$.

The calculation result of coefficient values $C_{k}$ and the corresponding frequency component $\omega_{n}+k \omega$ are summarized in Table 1 in which the theoretical spectrum of the response solution of parametric vibration can be predicted. The spectrum is schematically shown in Figure 5.

The response solution is very sensitive to $\omega$ while $\omega$ is closed to 0.5 . If $\omega$ is a little less than 0.5 , the high-order instability will be reflected in the solution (33). For example, in the case of $\omega=0.49845877$ in the above example, we get $C_{-4}=2825.8$. The result can be seen as a case of infinity, and it is governed by the left bias of higher-order instability. Simultaneously, the direct component $C_{-2}$ cannot be neglected. The detailed results are listed in Table 2 , and the theoretical response spectrum is drawn in Figure 6.

\section{Conclusion}

The response prediction of the single freedom of parametric vibration can be determined with a set of combinations 
TABLE 1: Calculation result of the value $C_{k}$ and corresponding frequency component $\omega_{n}+k \omega\left(\omega_{n}=1, \beta=0.3\right.$, and $\left.\omega=0.5\right)$.

\begin{tabular}{ccc}
\hline$K$ & Frequency $\omega_{n}+k \omega$ & $C_{k}$ value \\
\hline$\ldots$ & $\cdots$ & $\cdots$ \\
-8 & $-3.0 \omega_{n}$ & $-1.785 e-7$ \\
-7 & $-2.5 \omega_{n}$ & $-9.519 e-6$ \\
-6 & $-2 \omega_{n}$ & $-3.329 e-4$ \\
-5 & $-1.5 \omega_{n}$ & -0.0067 \\
-4 & $-\omega_{n}$ & -0.0551 \\
-3 & $-0.5 \omega_{n}$ & 0.0067 \\
-2 & 0 & 0.0218 \\
-1 & $0.5 \omega_{n}$ & -0.1522 \\
0 & $\omega_{n}$ & 1 \\
1 & $1.5 \omega_{n}$ & 0.1522 \\
2 & $2 \omega_{n}$ & 0.0076 \\
3 & $2.5 \omega_{n}$ & $2.1798 e-4$ \\
4 & $3.0 \omega_{n}$ & $4.086 e-6$ \\
$\ldots$ & $\ldots$ & $\ldots$ \\
\hline
\end{tabular}

TABLE 2: Calculation result of the value $C_{k}$ and corresponding frequency component $\omega_{n}+k \omega\left(\omega_{n}=1, \beta=0.3\right.$, and $\omega=$ 0.49845877).

\begin{tabular}{lcc}
\hline$K$ & Frequency $\omega_{n}+k \omega$ & $C_{k}$ value \\
\hline$\ldots$ & $\ldots$ & $\ldots$ \\
-8 & $\cong-3 \omega_{n}$ & 0.0096 \\
-7 & $\cong-2.5 \omega_{n}$ & 0.5090 \\
-6 & $\cong-2 \omega_{n}$ & 17.6233 \\
-5 & $\cong-1.5 \omega_{n}$ & 347.62 \\
-4 & $\cong-\omega_{n}$ & 2825.8 \\
-3 & $\cong-0.5 \omega_{n}$ & -579.18 \\
-2 & $\cong 0$ & 87.8963 \\
-1 & $\cong 0.5 \omega_{n}$ & -6.7887 \\
0 & $\omega_{n}$ & 1 \\
1 & $\cong 1.5 \omega_{n}$ & 6.7887 \\
2 & $\cong 2 \omega_{n}$ & 0.3413 \\
3 & $\cong 2.5 \omega_{n}$ & 0.0098 \\
4 & $\cong 3 \omega_{n}$ & 0.00018 \\
$\ldots$ & $\ldots$ & $\ldots$ \\
\hline
\end{tabular}

of harmonic components related to $\omega_{n}$ and $\omega$. Once the coefficients $C_{0}$ and $D_{0}$ are given, whether the general case or the special case of $\omega=2 \omega_{n} / q$, the harmonic components coefficients $C_{k}$ and $D_{k}$ can be fully determined by a set of series. On the coefficients $C_{k}$ and $D_{k}$, the corresponding theoretical spectrum can be drawn; it will be very useful in identifying and predicting the characteristics in the mechanical system on a model of parametric vibration, especially in the case of $\omega=2 \omega_{n} / q$.

The instability region can be determined when the frequency factor $\alpha_{k}$ is equal to zero. The investigation result matches the case of Hill's instability boundary curve. The harmonic resonance in the high-order instability region has

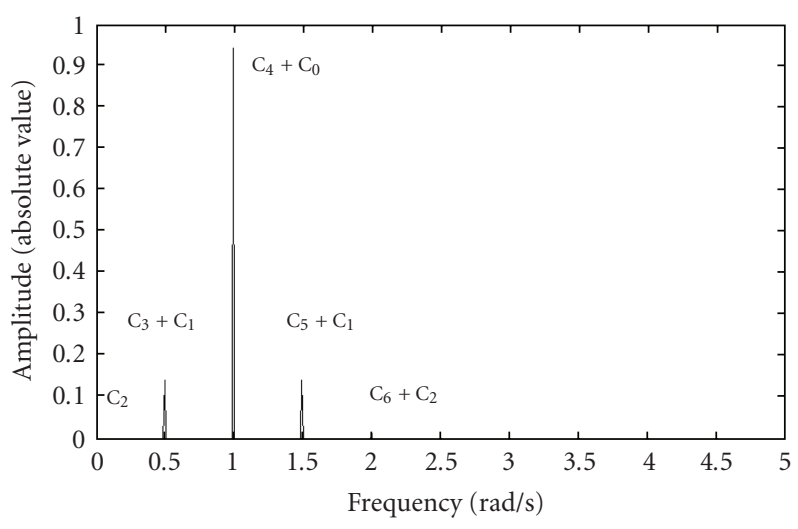

FIGURE 5: Spectrum of the parametric vibration response solution $\left(\omega_{n}=1, \beta=0.3, \omega=0.5\right)$.

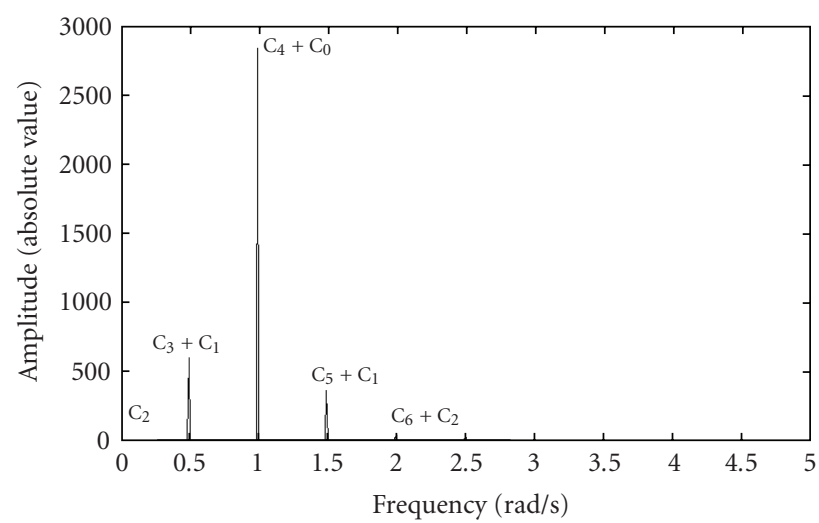

FIGURE 6: Spectrum of the parametric vibration response solution in the case of higher-order harmonic resonance $\left(\omega_{n}=1, \beta=0.3\right.$, and $\omega=0.49845877)$.

three features, which are left bias, region overlap, and cross direction.

The presented concept of modulation feedback reflects the physical inherency in the parametric vibration system, though it comes from the scope of a modulation system. This can become an available tool for us to analyze the response in the parametric vibration. Therefore, it is expected to obtain a response prediction of parametric vibration with an external force excitation and of a multiple freedom of parametric vibration, and so on.

\section{Acknowledgment}

The authors wish to acknowledge the support of the 211 Leading Academic Discipline Project of Shanghai University.

\section{References}

[1] V. A. Yakubovitch and V. M. Starzhinskii, Linear Differential Equation with Periodic Coefficients, John Wiley \& Sons, New York, NY, USA, 1975.

[2] A. H. Nayfeh, Problems in Perturbation, John Wiley \& Sons, New York, NY, USA, 1985. 
[3] G. H. Gaonkar, D. S. Simha Prasad, and D. Sastry, "On computing Floquet transition matrices of rotorcraft," Journal of the American Helicopter Society, vol. 26, no. 3, pp. 56-61, 1981.

[4] S. C. Sinha and D. H. Wu, "An efficient computational scheme for the analysis of periodic systems," Journal of Sound and Vibration, vol. 151, no. 1, pp. 91-117, 1991.

[5] S. C. Sinha, D. H. Wu, V. Juneja, and P. Joseph, "Analysis of dynamic systems with periodically varying parameters via Chebyshev polynomials," Transaction of the ASME, Journal of Vibration and Acoustics, vol. 115, no. 1, pp. 96-102, 1993.

[6] P. P. Friedmann, "Numerical methods for determining the stability and response of periodic systems with applications to helicopter rotor dynamics and aeroelasticity," Computers and Mathematics with Applications, vol. 12, no. 1, pp. 131-148, 1986.

[7] H. D’Angelo, Linear Time-Varying System: Analysis and Synthesis, Allyn and Bacon, Boston, Mass, USA, 1970.

[8] C. S. Hsu, "On approximating a general linear periodic system," Journal of Mathematical Analysis and Applications, vol. 45, no. 1, pp. 234-251, 1974.

[9] J. A. Richards, Analysis of Periodically Time-Varying System, Springer, Berlin, Germany, 1983.

[10] R. Gasch, "Dynamic behavior of a simple rotor with crosssectional crack," IMechE, vol. 170, pp. 123-128, 1976.

[11] A. H. Nayfeh and D. T. Mook, Non-Linear Oscillations, John Wiley \& Sons, New York, NY, USA, 1979. 

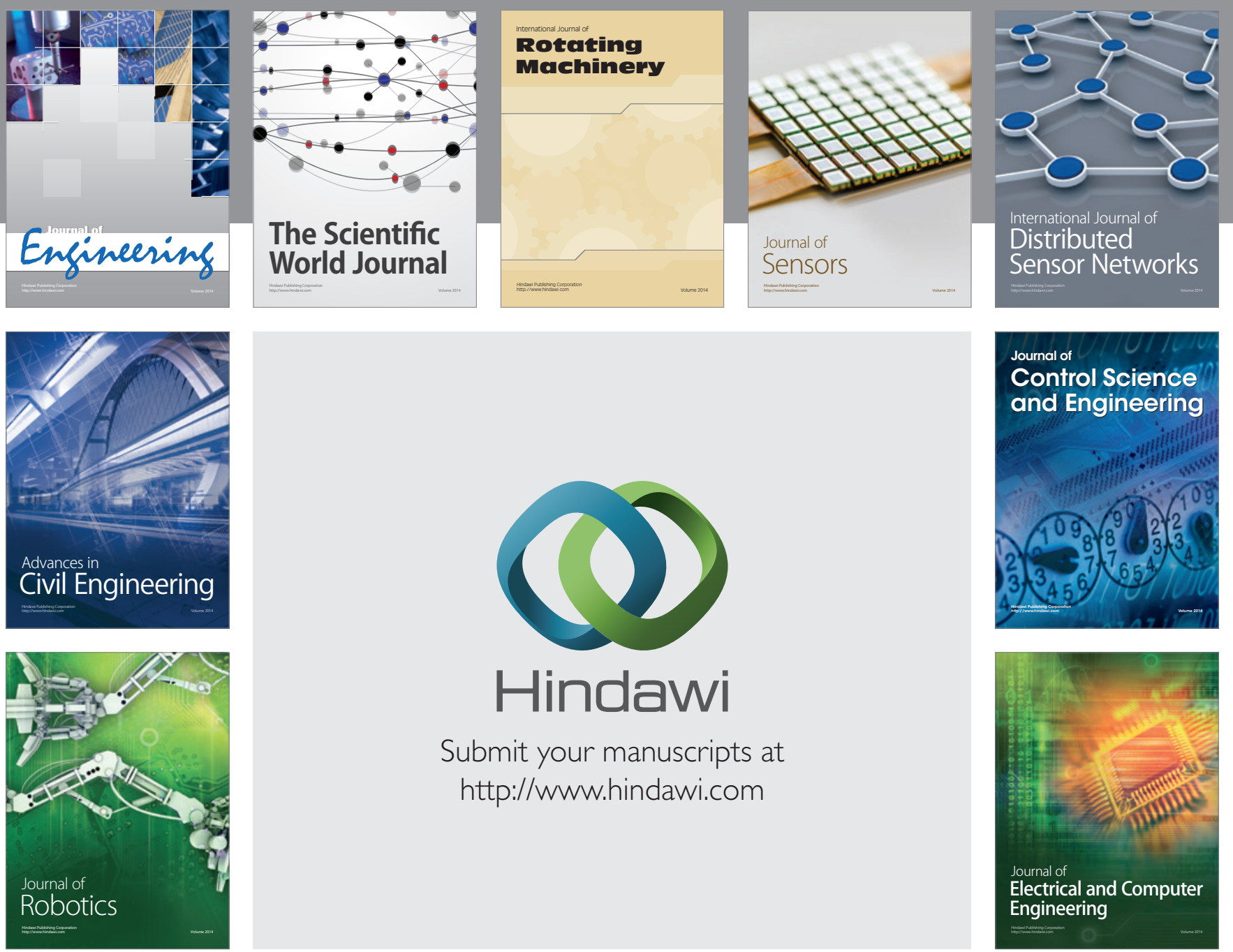

Submit your manuscripts at

http://www.hindawi.com
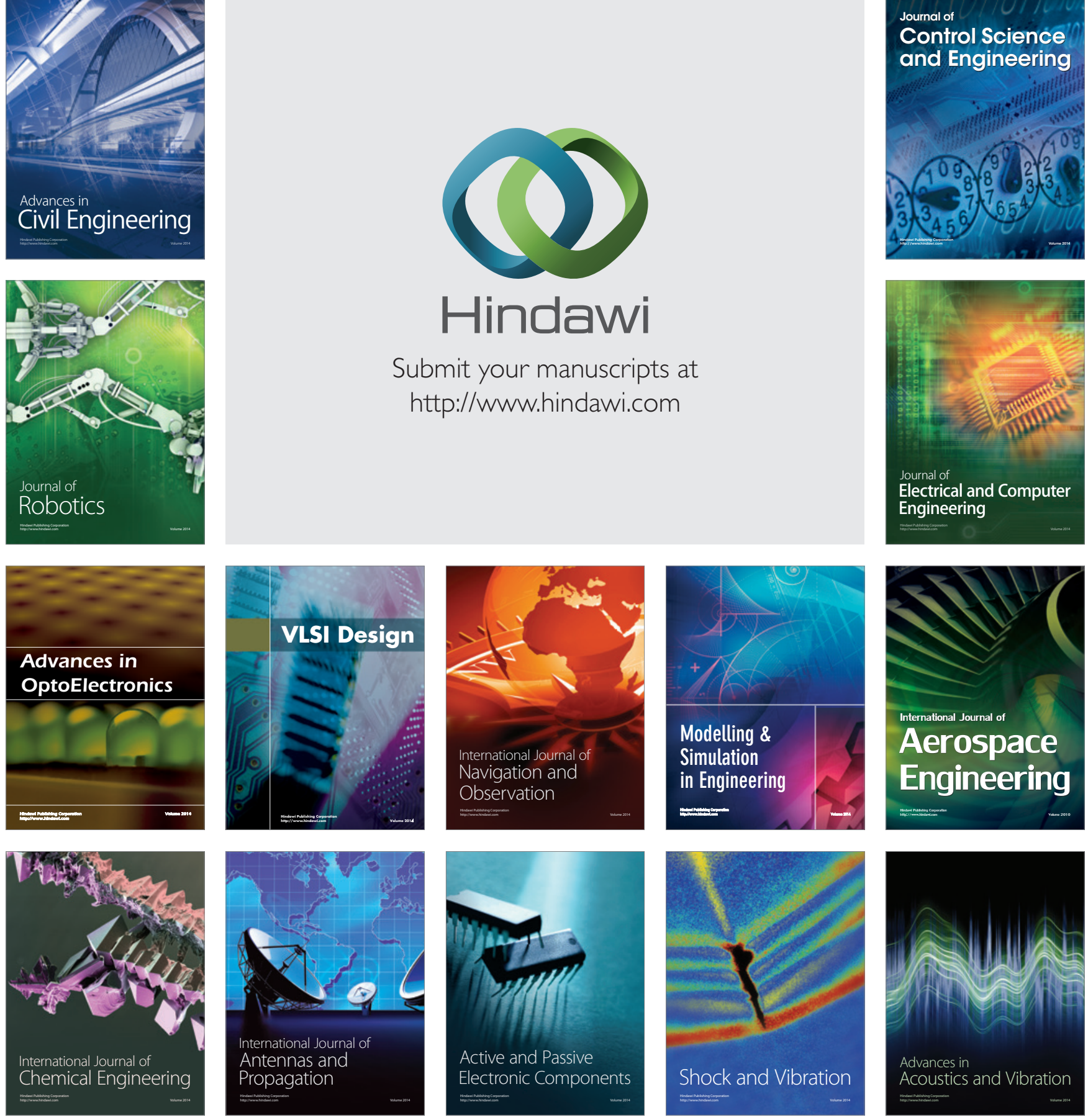